\title{
Variable stars in nearby galaxies ${ }^{\star, \star \star}$
}

\section{Fields C and D of IC 1613}

\author{
L. Mantegazza, E. Antonello, D. Fugazza, M. Bossi, and S. Covino \\ Osservatorio Astronomico di Brera, Via E. Bianchi 46, 23807 Merate, Italy
}

Received 23 October 2000 / Accepted 9 November 2000

\begin{abstract}
The nearby galaxies IC 1613 and NGC 6822 were observed over four years to detect short period Cepheids and to obtain good light curves for Fourier decomposition. The program was carried out with a relatively small telescope, the Dutch $0.9 \mathrm{~m}$ at ESO-La Silla, and the observations were performed without filter (white light), or $W h$-band. In this paper we present the results of the observations of Fields C and D in IC $1613\left(3{ }^{\prime} 8 \times 33^{\prime} 8\right.$ each). The analysis of the light curves of 3297 stars in the two fields has allowed the detection of 92 variable stars. A total of 34 Population I Cepheids have been found, while only 7 of these objects were previously known in these fields. Most of the stars have short periods and some of them are first overtone mode pulsators. Among the other variable stars there are one eclipsing binary and five W Vir candidates. A nova event was observed in Field D.
\end{abstract}

Key words. stars: oscillations; Cepheids; novae - stars: variables: general - galaxies: individual: IC 1613 galaxies: stellar content

\section{Introduction}

Cepheids are the primary calibrator for the extragalactic distance scale, and they are useful also for testing theories concerning the internal constitution of stars and stellar evolution. The study of Cepheids in nearby galaxies is important for understanding the effects of different metallicities and corresponding mass-luminosity relations on the pulsational characteristics of these variables. This is done through the detection of structures in the Fourier parameter-period diagrams of fundamental, first overtone and possibly double-mode and second overtone mode Cepheids, and their comparison with galactic Cepheids and model predictions. Large samples of Cepheids have been discovered in the Magellanic Clouds by microlensing programs (MACHO, EROS, OGLE; see e.g. Welch et al. 1997; Beaulieu \& Sasselov 1997; Udalski et al. 1999), and in M 31 and M 33 by the DIRECT project (e.g. Kaluzny et al. 1999). The purpose of our project was to obtain good light curves of Cepheids in the galaxies IC 1613 and NGC 6822, to extend the comparison of the characteristics of these stars. In order to make best use of the telescope time and to reach fainter luminosities, our

Send offprint requests to: L. Mantegazza,

e-mail: luciano@merate.mi.astro.it

* Based on observations collected at ESO-La Silla.

** Tables 3 and 4 are also available at the CDS via anonymous ftp to cdsarc.u-strasbg.fr $(130.79 .128 .5)$ or via http://cdsweb.u-strasbg.fr/cgi-bin/qcat?J/A+A/367/759 strategy was to observe in white light, i.e. without filter (Wh photometry). The results of the survey for variable stars in Field A of IC 1613 were reported in Paper I of this series (Antonello et al. 1999a) and the properties of Population I and II Cepheids observed in that field were discussed in Paper II (Antonello et al. 1999b). Paper III (Antonello et al. 2000b) presented the variable stars detected in Field B. Another paper (Antonello et al. 2000a) analyzed and compared the light curves of Cepheids with periods between 5 and 13 days in IC 1613 with corresponding objects in the Galaxy and in the Magellanic Clouds. Theoretical models are not able to reproduce correctly the light curves of Cepheids in the Magellanic Clouds, and the seriousness of this problem is essentially confirmed by the stars of IC 1613. In other words, a large discrepancy exists between theory and observations for low metallicity Cepheids. An introduction and description of the project on IC 1613 is reported in Paper I, and the methods and techniques are in further details discussed in Paper III. In the present paper we report about the results of the analysis of Fields C and D.

\section{Observations}

The observations of the irregular galaxy IC $1613[\alpha=$ $1^{\mathrm{h}} 04^{\mathrm{m}} 50^{\mathrm{s}}(2000), \delta=+2^{\circ} 08^{\prime}(2000), l=130^{\circ}, b=-61^{\circ}$, were performed with the direct CCD camera at the Dutch $0.91 \mathrm{~m}$ telescope of La Silla (ESO) during six runs from October 1995 to October 1998. The CCD detector was the 
Table 1. Log of observations of Field C of IC 1613

\begin{tabular}{|c|c|c|c|c|c|c|c|c|c|}
\hline & date & $\begin{array}{l}\text { Hel.J.D. } \\
2450000 .+\end{array}$ & Airmass & $\begin{array}{l}F W H M \\
\left({ }^{\prime \prime}\right)\end{array}$ & & date & $\begin{array}{l}\text { Hel.J.D. } \\
2450000 .+\end{array}$ & Airmass & $\begin{array}{l}F W H M \\
\left({ }^{\prime \prime}\right)\end{array}$ \\
\hline 1 & 1995 Oct. 20 & 011.83 & 2.11 & 1.8 & 27 & 1997 Oct. 30 & 752.56 & 1.38 & 1.9 \\
\hline 2 & 1995 Oct. 22 & 013.65 & 1.18 & 1.7 & 28 & & 752.66 & 1.18 & 1.7 \\
\hline 3 & 1995 Oct. 23 & 014.80 & 1.62 & 1.9 & 29 & 1997 Oct. 31 & 753.55 & 1.38 & 1.6 \\
\hline 4 & 1995 Oct. 25 & 016.71 & 1.21 & 1.7 & 30 & & 753.70 & 1.27 & 1.6 \\
\hline 5 & 1995 Oct. 26 & 017.74 & 1.33 & 1.9 & 31 & & 753.71 & 1.30 & 1.7 \\
\hline 6 & 1996 Oct. 13 & 370.64 & 1.23 & 1.7 & 32 & 1997 Nov. 01 & 754.56 & 1.31 & 2.2 \\
\hline 7 & & 370.77 & 1.36 & 1.7 & 33 & & 754.66 & 1.28 & 2.0 \\
\hline 8 & 1996 Oct. 14 & 371.80 & 1.28 & 1.6 & 34 & 1998 Jul. 25 & 1020.84 & 1.28 & 1.9 \\
\hline 9 & & 371.76 & 1.35 & 1.4 & 35 & & 1020.85 & 1.24 & 1.9 \\
\hline 10 & 1996 Oct. 15 & 372.76 & 1.31 & 1.6 & 36 & 1998 Jul. 26 & 1021.85 & 1.25 & 1.6 \\
\hline 11 & 1996 Oct. 16 & 373.64 & 1.20 & 1.5 & 37 & 1998 Jul. 30 & 1025.86 & 1.22 & 2.1 \\
\hline 12 & & 373.79 & 1.55 & 1.6 & 38 & 1998 Jul. 31 & 1026.85 & 1.23 & 1.6 \\
\hline 13 & 1996 Oct. 17 & 374.62 & 1.25 & 1.5 & 39 & 1998 Sep. 30 & 1087.76 & 1.20 & 1.5 \\
\hline 14 & & 374.75 & 1.32 & 1.4 & 40 & 1998 Oct. 01 & 1088.80 & 1.35 & 1.8 \\
\hline 15 & 1996 Oct. 18 & 375.57 & 1.50 & 1.5 & 41 & 1998 Oct. 02 & 1089.71 & 1.17 & 1.5 \\
\hline$V$ & & 375.69 & 1.17 & 1.3 & 42 & 1998 Oct. 08 & 1095.66 & 1.21 & 1.7 \\
\hline$R$ & & 375.72 & 1.22 & 1.4 & 43 & 1998 Oct. 09 & 1096.68 & 1.17 & 1.7 \\
\hline 16 & 1996 Oct. 19 & 376.57 & 1.41 & 1.8 & 44 & 1998 Oct. 10 & 1097.60 & 1.39 & 1.7 \\
\hline 17 & & 376.69 & 1.20 & 1.5 & 45 & & 1097.76 & 1.18 & 1.5 \\
\hline 18 & 1996 Oct. 20 & 377.56 & 1.50 & 1.9 & 46 & 1998 Oct. 11 & 1098.60 & 1.38 & 1.6 \\
\hline 19 & & 377.69 & 1.19 & 1.5 & 47 & & 1098.70 & 1.17 & 1.5 \\
\hline 20 & 1996 Oct. 21 & 378.77 & 1.53 & 1.8 & 48 & 1998 Oct. 13 & 1100.60 & 1.41 & 1.8 \\
\hline 21 & 1997 Oct. 27 & 749.59 & 1.25 & 1.8 & 49 & & 1100.70 & 1.17 & 1.7 \\
\hline 22 & & 749.70 & 1.22 & 1.8 & 50 & 1998 Oct. 14 & 1101.70 & 1.17 & 1.8 \\
\hline 23 & 1997 Oct. 28 & 750.56 & 1.39 & 1.8 & 51 & 1998 Oct. 15 & 1102.65 & 1.21 & 1.5 \\
\hline 24 & & 750.66 & 1.18 & 1.8 & 52 & & 1102.75 & 1.25 & 1.6 \\
\hline 25 & 1997 Oct. 29 & 751.56 & 1.38 & 1.9 & 53 & 1998 Oct. 16 & 1103.67 & 1.18 & 1.5 \\
\hline 26 & & 751.66 & 1.17 & 1.9 & 54 & & 1103.77 & 1.33 & 1.6 \\
\hline
\end{tabular}

ESO chip No. 33, which is a TEK CCD with $512 \times 512$ pixels, pixel size of $27 \mu \mathrm{m}$ and spatial resolution of 0.44 , providing a small field of view of $3.77 \times 3 ! 77$. Given the size of the field of view the need to observe not too far from the meridian and to get two images of the same field in the same night, we were forced to limit our programme to four selected fields of IC 1613, whose locations are shown in Fig. 1 of Paper I. Most of the observations were performed without a filter in order to get the best photon statistics for the study of faint Cepheid light curves; a few images were taken in Johnson $V$ and $R$ filter for comparison with previous work. Bias and twilight flat field frames were gathered in each useful night. Fields C and D were observed for 35 and 38 nights, respectively, starting from October 1995, and a total of 54 and $57 \mathrm{Wh}$ images were collected. The complete log of observations is reported in Tables 1 and 2 for Fields $\mathrm{C}$ and D, respectively. The tables contain, for each image, the date of the beginning of the night, the heliocentric Julian date of midexposure, the airmass and the mean FWHM PSF value (which takes into account both seeing and possible non-perfect focussing of the telescope). Each $W h, V$ and $R$ image is the sum of two or three consecutive exposures totalling $1800 \mathrm{~s}$.

\section{Data reduction and analysis}

Bias subtraction and flat field corrections were performed using the IRAF package (Tody 1993). It was necessary to correct the images for the slightly different pointing of the telescope and orientation of the CCD camera in the different runs. Therefore they were shifted and rotated using the ESO/MIDAS command REBIN/ROTATE with respect to image No. 14 for Field C and image No. 24 for Field D; these images are the best and were used as templates. The stellar photometry was performed by means of the IRAF/DAOPHOT package (Stetson 1987; Davis 1994). First, a list of reliable stars was prepared by selecting the common objects in the best four images of each field, after having analyzed them as in Paper I. In this way 1841 and 1456 objects were kept as bona fide stars for Field C and D, respectively. The brightness of these stars was then measured in all frames with the technique of "fixed position photometry" described by Kaluzny et al. (1998). The stars of Field C have at most 54 measurements and those of Field D 57; some stars have fewer measurements either because they were close to the edge of the frame, and therefore were not observed in all of the runs, or because they were rejected by the ALLSTAR routine in some frames. The same reduction procedure was adopted 
Table 2. Log of observations of Field D of IC 1613

\begin{tabular}{|c|c|c|c|c|c|c|c|c|c|}
\hline & date & $\begin{array}{l}\text { Hel.J.D. } \\
2450000 .+\end{array}$ & Airmass & $\begin{array}{l}F W H M \\
\left({ }^{\prime \prime}\right)\end{array}$ & & date & $\begin{array}{l}\text { Hel.J.D. } \\
\text { 2450000.+ }\end{array}$ & Airmass & $\begin{array}{l}F W H M \\
\left({ }^{\prime \prime}\right)\end{array}$ \\
\hline 1 & 1995 Oct. 21 & 12.57 & 1.39 & 1.6 & 30 & 1996 Oct. 21 & 378.74 & 1.35 & 1.6 \\
\hline 2 & 1995 Oct. 22 & 13.71 & 1.24 & 1.5 & 31 & 1997 Oct. 27 & 749.65 & 1.17 & 1.8 \\
\hline 3 & 1995 Oct. 23 & 14.82 & 2.31 & 2.2 & 32 & & 749.75 & 1.49 & 1.6 \\
\hline 4 & & 14.83 & 2.65 & 2.2 & 33 & 1997 Oct. 28 & 750.59 & 1.22 & 1.9 \\
\hline 5 & 1995 Oct. 25 & 16.73 & 1.34 & 1.5 & 34 & & 750.69 & 1.21 & 1.9 \\
\hline 6 & 1995 Oct. 26 & 17.57 & 1.36 & 2.2 & 35 & 1997 Oct. 29 & 751.58 & 1.27 & 2.0 \\
\hline 7 & & 17.58 & 1.30 & 2.0 & 36 & & 751.68 & 1.21 & 1.9 \\
\hline 8 & 1996 ago 07 & 303.61 & 1.45 & 2.2 & 37 & 1997 Oct. 30 & 752.57 & 1.29 & 2.0 \\
\hline 9 & 1996 ago 08 & 304.73 & 1.19 & 1.9 & 38 & & 752.68 & 1.22 & 2.0 \\
\hline$V$ & & 304.68 & 1.19 & 1.5 & 39 & & 752.69 & 1.20 & 1.9 \\
\hline$R$ & & 304.70 & 1.17 & 1.7 & 40 & 1997 Oct. 31 & 753.58 & 1.27 & 1.9 \\
\hline 10 & 1996 ago 09 & 305.70 & 1.17 & 1.6 & 39 & 1997 Nov. 01 & 754.59 & 1.22 & 2.2 \\
\hline 11 & 1996 ago 11 & 307.62 & 1.26 & 2.0 & 40 & & 754.69 & 1.23 & 2.0 \\
\hline 12 & & 307.63 & 1.22 & 2.5 & 41 & 1998 Jul. 25 & 1020.93 & 1.21 & 1.9 \\
\hline 13 & & 307.71 & 1.17 & 1.9 & 42 & 1998 Jul. 26 & 1021.82 & 1.31 & 1.8 \\
\hline 14 & 1996 ago 12 & 308.65 & 1.25 & 2.3 & 43 & 1998 Jul. 30 & 1025.83 & 1.25 & 2.1 \\
\hline 15 & 1996 ago 13 & 309.64 & 1.20 & 1.9 & 44 & 1998 Jul. 31 & 1026.82 & 1.27 & 1.5 \\
\hline 16 & & 309.74 & 1.25 & 2.0 & 45 & 1998 Oct. 01 & 1088.72 & 1.17 & 1.5 \\
\hline 17 & 1996 Oct. 13 & 370.67 & 1.18 & 1.8 & 46 & 1998 Oct. 02 & 1089.68 & 1.18 & 2.1 \\
\hline 18 & & 370.80 & 1.55 & 1.6 & 47 & 1998 Oct. 10 & 1097.70 & 1.18 & 1.5 \\
\hline 19 & 1996 Oct. 14 & 371.64 & 1.20 & 1.4 & 48 & & 1097.80 & 1.47 & 1.9 \\
\hline 20 & & 371.79 & 1.48 & 1.5 & 49 & 1998 Oct. 11 & 1098.67 & 1.18 & 1.5 \\
\hline 21 & 1996 Oct. 15 & 372.78 & 1.47 & 1.6 & 50 & & 1098.72 & 1.34 & 1.5 \\
\hline 22 & 1996 Oct. 16 & 373.67 & 1.17 & 1.3 & 51 & 1998 Oct. 13 & 1100.63 & 1.25 & 1.9 \\
\hline 23 & 1996 Oct. 17 & 374.65 & 1.19 & 1.5 & 52 & & 1100.72 & 1.21 & 1.5 \\
\hline 24 & & 374.77 & 1.44 & 1.4 & 53 & 1998 Oct. 14 & 1101.73 & 1.24 & 1.8 \\
\hline 25 & 1996 Oct. 18 & 375.59 & 1.35 & 1.5 & 54 & 1998 Oct. 15 & 1102.63 & 1.25 & 1.5 \\
\hline 26 & 1996 Oct. 19 & 376.60 & 1.32 & 1.8 & 55 & & 1102.72 & 1.21 & 1.5 \\
\hline 27 & & 376.73 & 1.25 & 1.7 & 56 & 1998 Oct. 16 & 1103.62 & 1.25 & 1.5 \\
\hline 28 & 1996 Oct. 20 & 377.59 & 1.34 & 1.8 & 57 & & 1103.72 & 1.21 & 1.6 \\
\hline 29 & & 377.72 & 1.24 & 1.5 & & & & & \\
\hline
\end{tabular}

for both $W h$ and $V, R$ images. The transformation from instrumental $w h$ to "standard" $W h$ magnitudes was performed in the way described in Paper III, and using the same transformation equation. The standard deviations about the mean of the $W h$ measurements of all the stars studied in Fields C and D are shown in the two panels of Fig. 1 as a function of the mean stellar brightness.

The calibration of our $V$ and $R$ frames of Field D was performed in the same way as for Fields A and B, using as local standards the stars in common with Freedman (1988b, hereinfater FRE). This was not possible for Field C, because it does not overlap the FRE fields. Therefore, in order to get the colour information a different approach was required. We searched the ESO public archive and found $V$ and $I$ images of IC 1613 obtained on 1997 October 27 at the NTT with EMMI. These images provide an almost complete overlap of all our fields, and have $F W H M$ 's of $1.5^{\prime \prime}$. We also retrieved from the archive images of four Landolt (1992) standard star fields that were observed during that night with the same instrumentation, and we used them to calibrate the NTT images of
IC 1613. We carried out the photometry of these images in the same manner as we reduced our own data.

As discussed in Paper III, we also have calibrated BVI frames of the central region of IC 1613 obtained by Cole et al. (1999, hereafter CTG) using the WIYN telescope. Since these frames partially overlap Field D, we used them to obtain $B V I$ magnitudes for about 750 stars in it.

We compared the VI magnitudes of stars in common between the NTT and CTG images and found a satisfactory agreement: the zeropoints of the two systems differ by no more than a few hundredths of a magnitude. The comparison can be seen in Fig. 2. When both CTG and NTT data were available, we prefer the former because of their substantially better seeing $\left(0.6^{\prime \prime}\right.$ vs. $\left.1.5^{\prime \prime}\right)$.

\section{Variable stars}

The search for variable stars was performed with the same techniques described in Papers I and III. 52 probable variable stars were detected in Field C, and 40 in Field D. They include 34 Cepheids, 1 eclipsing binary, 47 long period variables, 7 other periodic variables, 1 nova and 


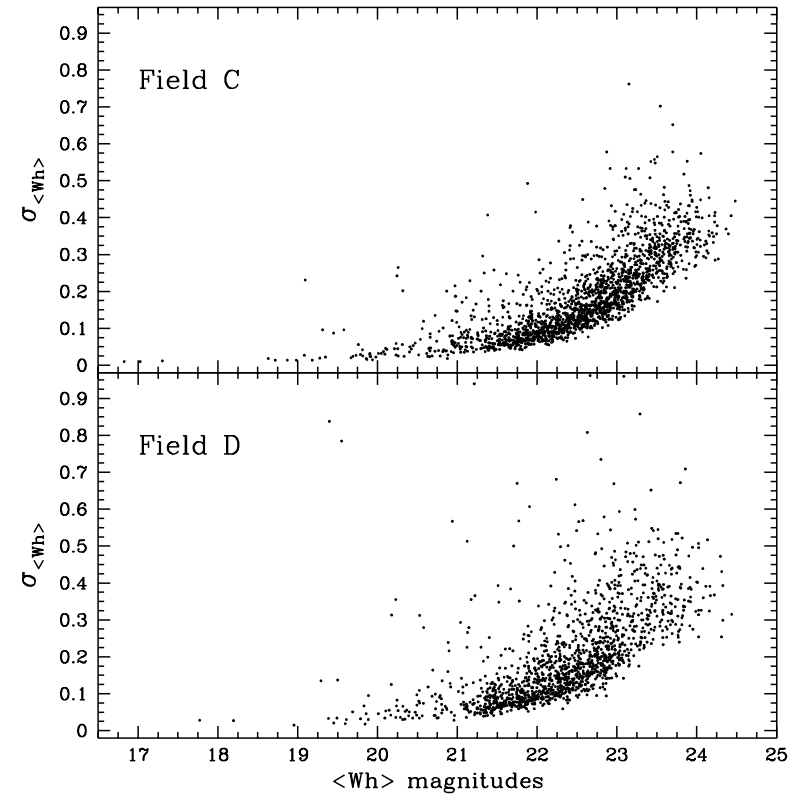

Fig. 1. Standard deviations of the $W h$ measurements of all the studied stars in Fields C (upper panel) and D (lower panel) vs. their average values. The brightest stars have measurements with accuracies of a few hundredths of a magnitude
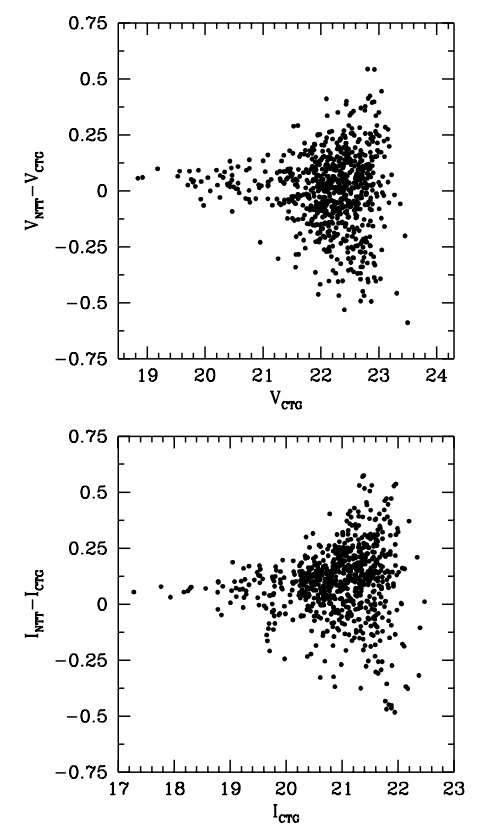

Fig. 2. Differences between standard $V$ (top panel) and $I$ (bottom panel) magnitudes of the objects in common between CTG and NTT fields as obtained by using FRE and Landolt calibrations

5 variable stars of uncertain type. The variables with available $V-I$ index are shown in the colour-magnitude diagrams reported in Fig. 3, where the dots indicate all the stars studied in the two fields and for which it was possible to derive the $V-I$ color. The star coordinates were derived with the same approach adopted for Fields $\mathrm{A}$ and $B$ and have an accuracy better than 1 arcsec. An atlas with the identification maps of the detected variables in the four Fields A, B, C and D will be published in a future paper.

\subsection{Cepheids}

The 34 detected Cepheids are listed in Table 3 and their light curves are displayed in Fig. 4; note that the magnitude scale is not the same in the different panels. The periods range from 0.6 to $23.5 \mathrm{~d}$ and the faintest object has a mean $W h$ magnitude of about 23.1, which should correspond to about $V \sim 23.3$. Just from a simple inspection it is possible to conclude that both fundamental and first overtone mode Cepheids have been detected; fundamental mode Cepheids have large amplitudes or asymmetric light curves, while first overtone mode stars have relatively small amplitudes and more symmetric light curves. We have used the Fourier parameters for a reliable discrimination of the pulsation mode, these parameters will be discussed in detail in the next paper. Table 3 reports for each star, its J2000 coordinates, period, mean Wh magnitude, Wh amplitude, probable pulsation mode, previous identification (if any). A few of these stars need some comments: V0314D: the light curve has the shape of a first overtone mode pulsator, but the colour is rather red $(V-R=0.74)$, and the star is rather bright. The more plausible explanation is that there is an unresolved close red star, a case similar to V2942B (Paper III). Unfortunately in this case the object is not included in CGT and NTT images, and a check of this hypothesis is not possible.

V0793D and V1307D: the periods of these stars are uncertain. Values of $0.571 \mathrm{~d}$ and $0.535 \mathrm{~d}$ could fit the data equally well, but in these cases the stars would not lie in the $P L$ relation defined by the other Cepheids.

$V 1345 D$ corresponds to $V 1894 B$, which is located in Field B (Paper III). It is very close to the border of Field D, so it was only present in a dozen frames. The light curve shown in Fig. 5 contains the data derived from both fields.

\subsection{The nova in Field $D$}

A nova $(V 0588 D)$ appeared in the frames taken in October 1996 as a bright object with $W h \sim 20$ and with a steadily decreasing brightness: its $W h$ luminosity dimmed by about $0.25 \mathrm{mag}$ in 9 days. In the images of the previous runs (October 1995 and August 1996) the object was not visible and the same is true for the images of the successive runs, meaning that in all these runs it was fainter than $W h \sim 24$. Hence the nova explosion occurred between August 14 and October 12, 1996. In Fig. 5 we show the $W h$ light curve observed in October 1996, and three images of the field of the nova taken just before (left), just after (centre), and one year after the event (right). The object is not visible both in the NTT and CTG frames, which were obtained in October 1997. The relevant data of this object are listed in Table 4. 


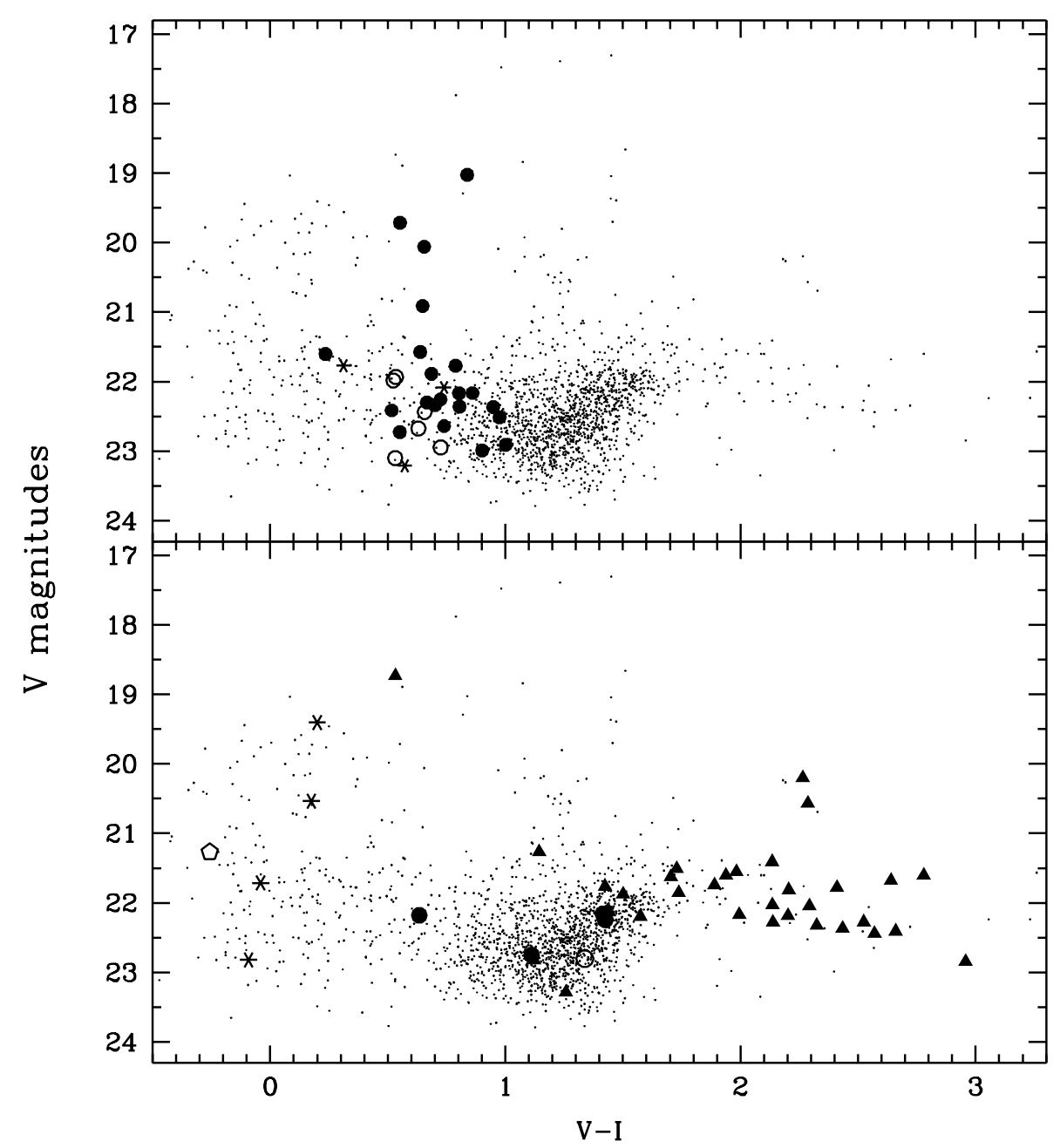

Fig. 3. Colour-magnitude $(V-I, V)$ diagram for the stars investigated in Fields C and D. Upper panel: open circles: first overtone mode Cepheids; filled circles: fundamental mode Cepheids; asterisks: Cepheids of uncertain mode. Lower panel: filled triangles: irregular and long period red variables; open pentagon: eclipsing binary; asterisks: blue variables; filled circles: W Vir candidates; open circle: red periodic variable

The discovery in this galaxy of two other novae was previously claimed. The first one (Sandage 1971) was detected by Baade on three plates in November 24, 1954 and it was not visible on a plate of November 21. No further plates were taken during that season and the star was not seen anymore. King et al. (1999) claimed the discovery of another nova in October 1999, in the context of the Lick Observatory Supernova Search. However we have shown (Fugazza et al. 2000) that such an object is not a nova but the red variable V2950B (see Paper III), which has a period of $631 \mathrm{~d}$ and a $W h$ amplitude of about $2.5 \mathrm{mag}$.

\subsection{Other variable stars}

The other variable stars are listed in Table 4 . In this table we give for each object: the identification; the J2000 coordinates; the mean $W h$ magnitude; the period, or the timescale if the variation is irregular; the observed $W h$ amplitude; the $V-I$ color index (or $V-R$, for some objects in Field D); the type of variability, LP: red irregular or long period variable, BV: blue variable, E: eclipsing variable,
RP: red periodic variable. Note that: a) the color index refers to a random phase of variation; b) in some cases the timescale is missing because, owing to the irregular variations, it was not possible to get a reliable value; in any case, since the spectral window contains a wide peak at low frequencies (see for instance Paper I), the value given for long period objects is only indicative.

There are 42 long period red stars, with timescales or periods ranging from 50 to $\sim 1000 \mathrm{~d}$, and $W h$ amplitudes between 0.2 and more than 1 magnitude. Selected light curves of these stars are shown in Figs. 6 and 7. The most conspicuous object is the bright star V1421D, which corresponds to Sandage's variable (SV19). According to Sandage (1971) it presents classification problems; the light curve does not repeat perfectly from cycle to cycle, but the period appears to be relatively stable, $446 \mathrm{~d}$. Our data confirm this period, and the $V-I$ value indicates that the star is very red. According to Sandage (1971) the $B$ amplitude is about $2.6 \mathrm{mag}$, while our data indicate a $W h$ amplitude of about $2.3 \mathrm{mag}$. The light curve is shown in one of the panels of Fig. 7. 

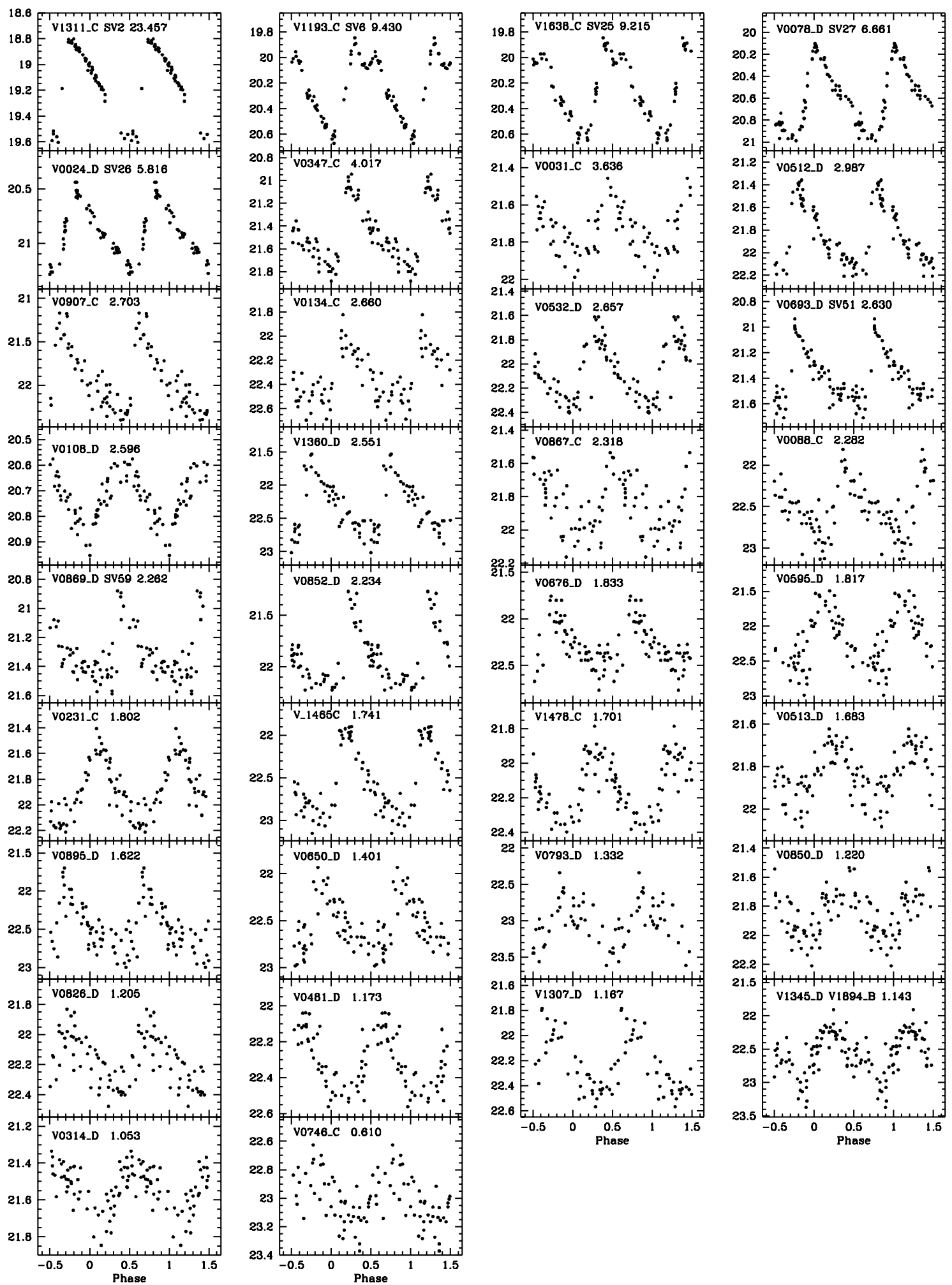

Fig. 4. Wh light curves of Cepheids in Fields C and D. For each star, the identification number and the period are reported. Note the different magnitude scales

$V 1018 D$ was already detected in Field B as $V 4541 B$ (Paper III). The star is very close to the border of Field D and it was possible to measure it only in 14 frames. The data are in agreement with those of Field B.
Four variables have a rather blue colour $(V-I<0.2)$. Three of them, V0138C, V0037D and V0119D, show variations on long timescales, while the variations of V0798C seem to have a period of $0.91 \mathrm{~d}$. 
Table 3. Cepheids in Fields C and D of IC1613

\begin{tabular}{|c|c|c|c|c|c|c|c|}
\hline Name & $\begin{array}{l}\alpha(2000) \\
{\left[\begin{array}{lll}\mathrm{h} & \mathrm{m} & \mathrm{s}\end{array}\right]}\end{array}$ & $\begin{array}{l}\delta(2000) \\
{\left[\begin{array}{lll}0 & , & \\
& & \end{array}\right]}\end{array}$ & $\begin{array}{l}P \\
{[\mathrm{~d}]}\end{array}$ & $\begin{array}{l}\overline{W h} \\
{[\mathrm{r}}\end{array}$ & $\begin{array}{l}\text { Ampl. } \\
\text { ag] }\end{array}$ & mode & other name \\
\hline V0031C & 010427.63 & 020435.1 & 3.636 & 21.79 & 0.32 & $\mathrm{~F}$ & \\
\hline V0088C & 010433.95 & $\begin{array}{lll}02 & 05 & 03.2\end{array}$ & 2.282 & 22.53 & 0.85 & $\mathrm{~F}$ & \\
\hline V0134C & 010428.29 & 020512.8 & 2.660 & 22.42 & 0.51 & $\mathrm{~F}$ & \\
\hline V0231C & 010429.64 & 020543.5 & 1.802 & 21.87 & 0.56 & 10 & \\
\hline V0347C & 010423.70 & 020607.6 & 4.017 & 21.46 & 0.77 & $\mathrm{~F}$ & \\
\hline V0746C & 010434.12 & 020808.8 & 0.610 & 22.99 & 0.35 & 10 & \\
\hline V0867C & 010436.04 & $\begin{array}{lll}02 & 0502.8\end{array}$ & 2.318 & 21.86 & 0.38 & $\mathrm{~F}$ & \\
\hline V0907C & 010423.98 & 020513.9 & 2.703 & 21.98 & 1.06 & $\mathrm{~F}$ & \\
\hline V1193C & 010433.73 & 020741.1 & 9.430 & 20.24 & 0.66 & $\mathrm{~F}$ & SV6 \\
\hline V1311C & 010432.25 & 020458.6 & 23.457 & 19.09 & 0.81 & $\mathrm{~F}$ & SV2 \\
\hline V1465C & 010429.30 & 020756.8 & 1.741 & 22.57 & 1.08 & $\mathrm{~F}$ & \\
\hline V1478C & 010423.09 & $\begin{array}{lll}02 & 08 & 08.8\end{array}$ & 1.701 & 22.13 & 0.44 & 10 & \\
\hline V1638C & 010434.57 & 020638.5 & 9.215 & 20.26 & 0.74 & $\mathrm{~F}$ & SV25 \\
\hline V0024D & 010443.81 & $\begin{array}{lll}02 & 01 & 04.1\end{array}$ & 5.816 & 20.89 & 0.72 & $\mathrm{~F}$ & SV26 \\
\hline V0078D & 010440.23 & 020123.8 & 6.661 & 20.58 & 0.88 & $\mathrm{~F}$ & SV27 \\
\hline V0108D & 010439.54 & 020133.5 & 2.596 & 20.73 & 0.30 & $10 ?$ & \\
\hline V0314D & 010439.54 & 020233.0 & 1.053 & 21.53 & 0.30 & 10 & \\
\hline V0481D & 010436.36 & 020306.8 & 1.173 & 22.34 & 0.38 & $1 \mathrm{O} ?$ & \\
\hline V0512D & 010440.03 & 020315.8 & 2.987 & 21.84 & 0.70 & $\mathrm{~F}$ & \\
\hline V0513D & 010439.27 & 020315.5 & 1.683 & 21.84 & 0.25 & 10 & \\
\hline V0532D & 010439.61 & 020318.7 & 2.657 & 22.09 & 0.59 & $\mathrm{~F}$ & \\
\hline V0595D & 010436.76 & 020330.2 & 1.817 & 22.17 & 0.97 & $\mathrm{~F}$ & \\
\hline V0650D & 010447.86 & 020347.1 & 1.401 & 22.57 & 0.68 & $\mathrm{~F}$ & \\
\hline V0676D & 010437.55 & 020342.9 & 1.833 & 22.30 & 0.54 & $\mathrm{~F}$ & \\
\hline V0693D & 010440.96 & 020347.8 & 2.630 & 21.38 & 0.53 & $\mathrm{~F}$ & SV51 \\
\hline V0793D & 010438.60 & 020358.4 & 1.332 & 23.14 & 0.70 & $\ldots$ & \\
\hline V0826D & 010441.18 & 020408.1 & 1.205 & 22.22 & 0.37 & 10 & \\
\hline V0850D & 010442.33 & 020417.2 & 1.220 & 21.87 & 0.40 & $1 \mathrm{O} ?$ & \\
\hline V0852D & 010439.24 & 020406.7 & 2.234 & 21.89 & 0.75 & $\mathrm{~F}$ & \\
\hline V0869D & 010441.18 & 020410.4 & 2.262 & 21.33 & 0.50 & $\mathrm{~F}$ & SV59 \\
\hline V0895D & 010436.07 & 020410.3 & 1.622 & 22.49 & 0.85 & $\mathrm{~F}$ & \\
\hline V1307D & 010438.46 & 020408.9 & 1.167 & 22.26 & 0.52 & $\ldots$ & \\
\hline V1345D & 010448.61 & 020445.2 & 1.143 & 22.57 & 0.72 & $\mathrm{~F}$ & V1894B \\
\hline V1360D & 010442.12 & 020412.9 & 2.551 & 22.33 & 1.16 & $\mathrm{~F}$ & \\
\hline
\end{tabular}
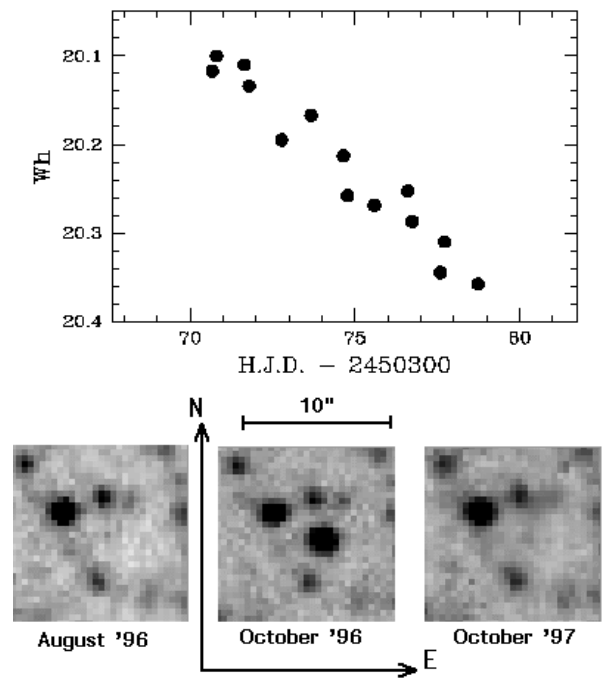

Fig. 5. Upper part: light curve of the nova ( $\equiv V 0588 D)$ observed in October 1996. Images of the nova's field just before the event (August 1996; left frame), just after the event (October 1996, central frame), and one year later (right frame)
The Population II Cepheids (or W Vir stars) should have colour indices not very different from those of Population I Cepheids, since the latter are rather metalpoor; moreover, for a given luminosity, Population II Cepheids have a much longer period. On these bases we have identified 5 possible candidates: $V 0043 C$, V0234C, V0765C, V0966C and V1202C. Their light curves are shown in Fig. 8. For V0043C the color information is missing; for $V 0966 C$ the color is rather red, and the amplitude is rather small. The light curve of this star could suggest the presence of a second period, but a specific search did not give positive results. The measured color of $1202 \mathrm{C}$ is rather red too, but it corresponds to the minimum light and the amplitude is larger than in the previous case. The amplitude of variation of $V 0765 C$ is rather small and the phasing of the data is clumpy, making its period uncertain.

Only one eclipsing variable has been confidently detected in Field C ( V0561C) and none in Field D. V0561C has a rather blue colour, a rather short period and shows two similar minima. The period reported in Table 4 is 
Table 4. Other variable stars in Fields $\mathrm{C}$ and D of IC1613. An asterisk indicates a $V-R$ value instead of $V-I$

\begin{tabular}{|c|c|c|c|c|c|c|c|}
\hline Name & $\begin{array}{l}\alpha(2000) \\
{\left[\begin{array}{lll}\mathrm{h} & \mathrm{m} & \mathrm{s}\end{array}\right]}\end{array}$ & $\begin{array}{l}\delta(2000) \\
{\left[\begin{array}{lll} & & \\
& & \end{array}\right]}\end{array}$ & $\begin{array}{l}\overline{W h} \\
{[\mathrm{mag}]}\end{array}$ & $\begin{array}{l}T \\
{[\mathrm{~d}]}\end{array}$ & $\begin{array}{r}\text { Ampl } \\
{[\mathrm{n}}\end{array}$ & $\begin{array}{l}V-I \\
\mathrm{~g}]\end{array}$ & Type \\
\hline V0043C & 010434.00 & 020444.6 & 22.59 & 9.412 & 0.50 & - & W Vir? \\
\hline V0130C & 010434.75 & $0205 \quad 17.2$ & 22.49 & 1.017 & 0.52 & 1.34 & $\mathrm{RP}$ \\
\hline V0138C & 010436.21 & 020521.1 & 22.77 & $23 ?$ & 0.9 & -.09 & $\mathrm{BV}$ \\
\hline V0145C & 010428.30 & 020517.2 & 21.59 & 142 & 0.45 & 2.00 & LP \\
\hline V0169C & 010426.77 & 020521.3 & 22.81 & 284 & 0.75 & 1.26 & LP \\
\hline V0234C & 010430.52 & 020544.5 & 22.56 & 9.062 & 0.40 & 1.11 & W Vir \\
\hline V0266C & 010434.16 & 020556.4 & 21.08 & - & 0.25 & 2.52 & LP \\
\hline V0285C & 010435.43 & 020601.9 & 20.94 & 208 & 0.35 & 2.07 & LP \\
\hline V0354C & 010426.02 & 020610.2 & 21.16 & 157 & 0.55 & 2.20 & LP \\
\hline V0364C & 010429.38 & 020615.7 & 21.40 & 680 & 0.70 & 2.32 & LP \\
\hline V0415C & 010436.04 & 020637.8 & 21.04 & 560 & 0.25 & 1.73 & LP \\
\hline V0491C & 010428.14 & 020659.3 & 21.17 & 78.84 & 0.45 & 1.74 & LP \\
\hline V0561C & 010426.45 & 020713.1 & 21.25 & 1.107 & 0.50 & -.26 & $\mathrm{E}$ \\
\hline V0567C & 010425.54 & 020713.8 & 23.08 & 19.317 & 1.0 & - & LP \\
\hline V0638C & 010428.08 & 020734.8 & 21.21 & 17.401 & 0.4 & 1.42 & LP \\
\hline V0762C & 010427.34 & 020807.0 & 21.47 & 70.22 & 0.20 & - & LP \\
\hline V0765C & 010433.60 & 020812.1 & 22.09 & 27.78 & 0.20 & 0.63 & W Vir? \\
\hline V0774C & 010433.57 & 020816.4 & 21.06 & 242 & 0.40 & 1.98 & $\mathrm{LP}$ \\
\hline V0798C & 010433.28 & 020638.5 & 21.65 & 0.909 & 0.35 & -.04 & BV \\
\hline V0825C & 010422.89 & 020429.3 & 21.14 & - & 0.25 & 1.14 & $\operatorname{Irr}$ \\
\hline V0833C & 010427.52 & 020437.5 & 21.01 & 81.79 & 0.20 & 2.29 & LP \\
\hline V0844C & 010428.74 & 020443.6 & 20.98 & 230 & 0.45 & 2.41 & LP \\
\hline V0860C & 010435.91 & 020458.2 & 22.58 & 18.746 & 0.50 & 1.12 & LP \\
\hline V0861C & 010431.13 & 020456.4 & 22.40 & 39.63 & 0.45 & - & LP \\
\hline V0869C & 010424.55 & 020454.2 & 20.32 & 428 & 0.65 & 2.78 & LP \\
\hline V0966C & 010434.63 & 020557.4 & 21.81 & 27.64 & 0.20 & 1.42 & W Vir? \\
\hline V0984C & 010425.80 & 020601.4 & 20.99 & 162 & 0.25 & 2.66 & LP \\
\hline V1074C & 010428.13 & 020640.7 & 20.95 & 640 & 0.20 & 1.94 & LP \\
\hline V1077C & 010430.68 & 020643.0 & 21.23 & 245 & 0.70 & 2.14 & LP \\
\hline V1090C & 010433.68 & 020650.9 & 21.11 & 154 & 0.15 & 1.70 & LP \\
\hline V1202C & 010429.50 & 020739.9 & 21.70 & 18.260 & 0.50 & 1.43 & W Vir? \\
\hline V1205C & 010430.20 & 020742.5 & 21.33 & 295 & 1.0 & - & LP \\
\hline $\mathrm{V} 1450 \mathrm{C}$ & 010433.00 & 020741.7 & 21.06 & 58.71 & 0.20 & 1.89 & LP \\
\hline V1578C & 010422.13 & 020723.8 & 20.97 & $>1000$ & 0.45 & 2.14 & LP \\
\hline V1594C & 010434.88 & 020750.9 & 21.28 & 47.50 & 0.20 & 2.96 & LP \\
\hline V1598C & 010427.64 & 020755.5 & 20.87 & 610 & 0.60 & - & LP \\
\hline $\mathrm{V} 1642 \mathrm{C}$ & 010435.49 & 020650.2 & 21.38 & 400 & $>1$ & 2.55 & LP \\
\hline V1649C & 010434.74 & 020713.1 & 19.31 & 530 & 0.25 & 2.66 & LP \\
\hline V1667C & 010427.07 & 020814.2 & 21.02 & - & 0.20 & 0.94 & Irr \\
\hline V0023D & 010448.48 & 020107.6 & 20.68 & 420 & 0.30 & $0.55^{*}$ & LP \\
\hline V0025D & 010445.26 & 020205.6 & 21.90 & 26.78 & 0.40 & $0.59^{*}$ & $?$ \\
\hline V0037D & 010440.78 & 020109.2 & 19.29 & 62.71 & 0.35 & 0.20 & BV \\
\hline V0119D & 010435.98 & 020133.5 & 20.52 & - & 0.20 & 0.18 & $\mathrm{BV}$ \\
\hline V0140D & 010436.39 & 020139.0 & 22.28 & 310 & 0.50 & $0.60^{*}$ & LP \\
\hline V0170D & 010436.81 & 020148.8 & 21.62 & - & 0.30 & 0.75 & Irr \\
\hline V0202D & 010443.29 & 020107.7 & 21.22 & 270 & 1.0 & $1.14^{*}$ & LP \\
\hline V0341D & 010449.13 & 020245.1 & 20.91 & 260 & 0.40 & $0.96^{*}$ & LP \\
\hline V0365D & 010441.76 & 020246.7 & 21.45 & $23 ?$ & 0.40 & 1.50 & LP \\
\hline V0417D & 010443.71 & 020256.5 & 20.93 & 190 & 0.30 & $0.69^{*}$ & LP \\
\hline V0421D & 010447.25 & 020259.5 & 21.05 & 380 & 0.45 & $0.74^{*}$ & LP \\
\hline V0520D & 010449.21 & 020324.2 & 20.88 & 570 & 0.45 & 2.43 & LP \\
\hline V0582D & 010450.62 & 020339.9 & 20.52 & 910 & 0.30 & 2.14 & LP \\
\hline V0588D & 010444.06 & 020334.3 & 22.02 & - & $>4$ & - & Nova \\
\hline V0761D & 010442.60 & 020357.9 & 21.68 & 14.189 & 0.40 & 1.44 & LP \\
\hline V0868D & 010450.33 & 020418.0 & 22.37 & 165 & 1.0 & $0.60^{*}$ & LP \\
\hline V0921D & 010441.38 & 020426.5 & 19.51 & 144 & 0.40 & 2.29 & LP \\
\hline V1018D & 010450.37 & 020445.2 & 20.17 & 51.84 & 0.35 & 2.64 & LP V4541B \\
\hline V1421D & 010436.34 & 020334.4 & 19.40 & 445 & 2.30 & 0.53 & LP SV19 \\
\hline
\end{tabular}




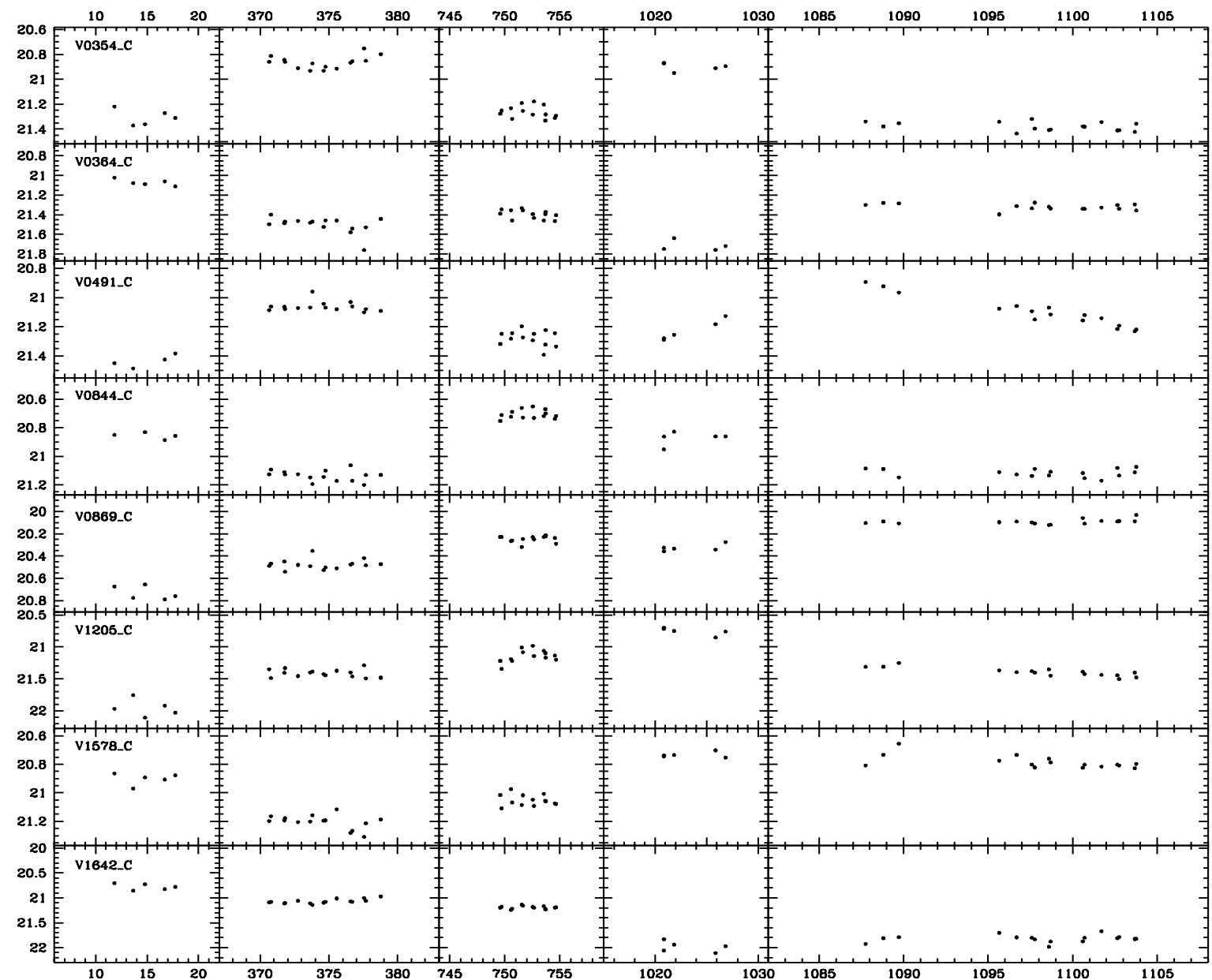

Fig. 6. Wh light curves of a sample of long period and irregular variables in Field C. Note the different magnitude scales

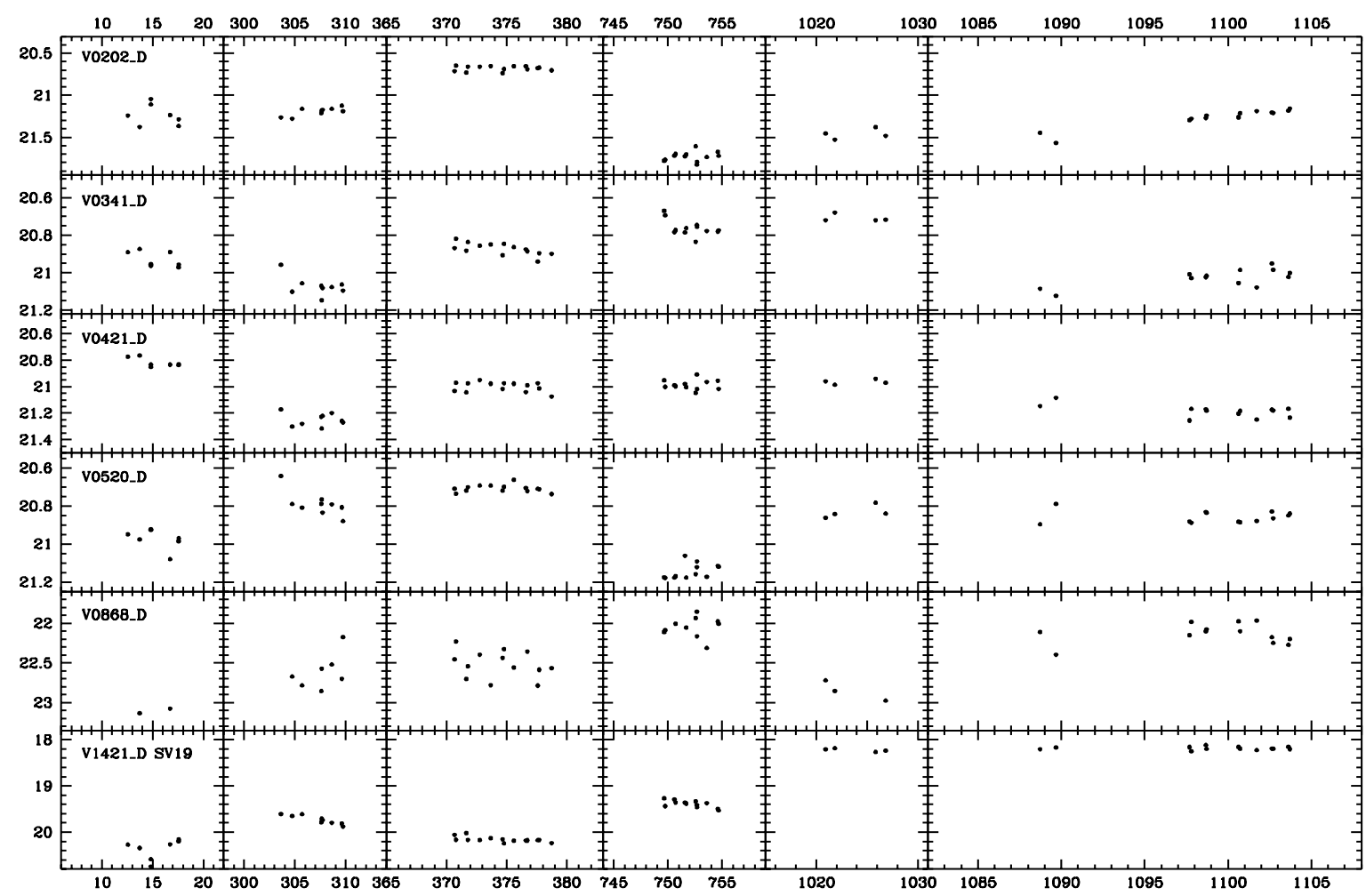

Fig. 7. Wh light curves of a sample of long period and irregular variables in Field D. Note the different magnitude scales 

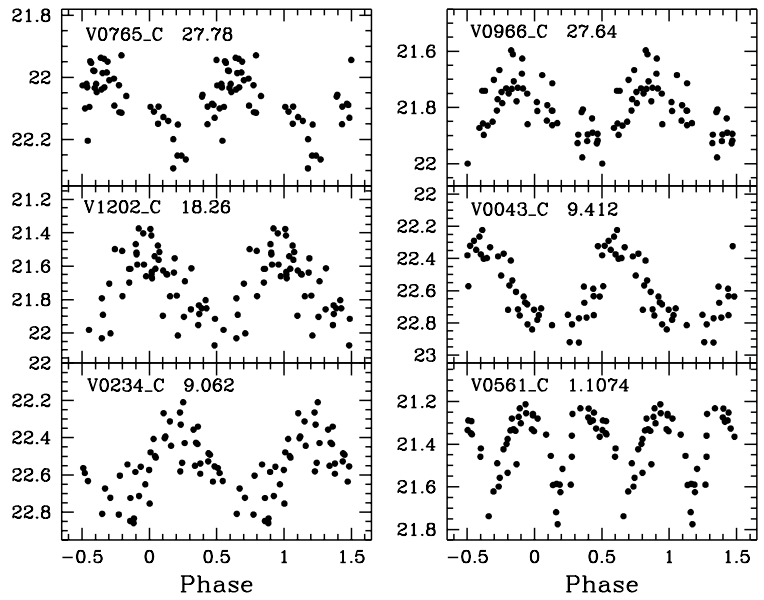

Fig. 8. Phased light curves of the five W Vir candidates and of the eclipsing binary (V0561C)

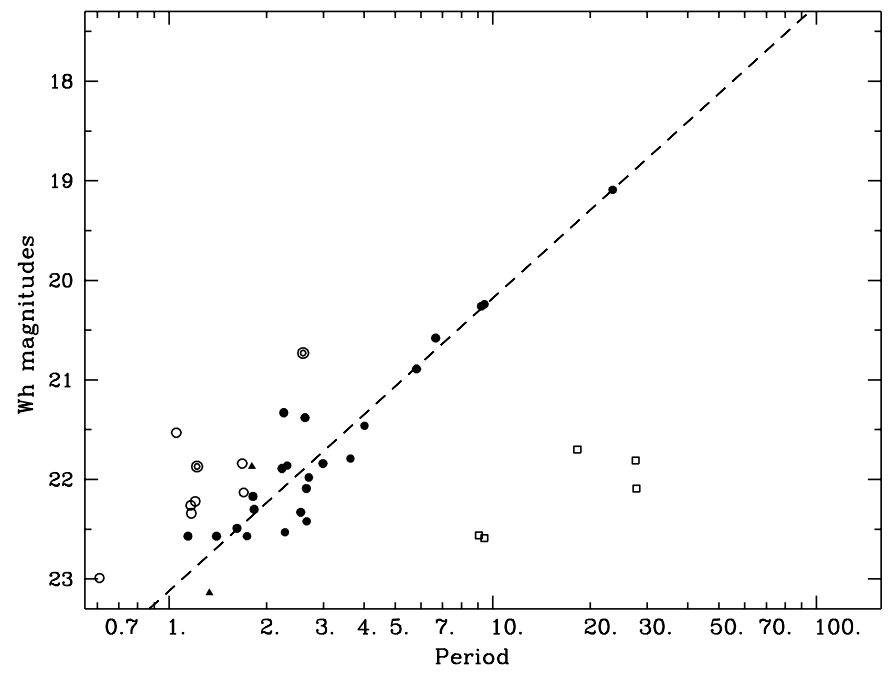

Fig. 9. $P L$ diagram for Populations I and II Cepheids in Fields C and D of IC 1613. Dots: fundamental mode Cepheids; open circles: first overtone mode Cepheids; Filled triangles: Cepheids with uncertain pulsation mode; annuli: first overtone mode candidates; open squares: W Vir candidates. The dashed line is the statistical relation obtained for Pop. I fundamental mode Cepheids of Fields A and B

twice the separation between minima, and the magnitude corresponds to the luminosity at maximum. The light curve is shown in the bottom right panel of Fig. 8.

The light curve of $V 0130 C$ and its position in the $P-L$ diagram suggests that it could be a first-overtone Cepheid. However its colour is too red and we are not able to find an explanation for it. Therefore we classified the star as a red periodic variable (RP).

Finally, V0025D is certainly variable and with a probable period of about 26.8 days, but its light curve does not bear any resemblance with those of pulsating variables and therefore its classification is uncertain.

\subsection{Previously known variable stars}

The following variable stars, previously discovered by Sandage (1971), are present in the two fields: SV2, SV6,
$S V 25$ and $S V 50$ in Field C and SV19, SV26, SV27, SV51, $S V 59$ and $S V 63$ in Field D. All of them were independently detected as variable stars by us with the exception of $S V 50$ and $S V 63$.

$S V 50$ was classified by Sandage (1971) as a reddish, though not extreme, long period variable with a time scale of about $2600 \mathrm{~d}$ and a $B$ amplitude of about 0.5 mag. It corresponds to the object $V 1554 C$, which is constant within \pm 0.05 mag. Our measurements of CTG frames give $V=20.77, B-V=-0.02$ and $V-I=0.15$. The $B$ magnitude is within the extrema given by Sandage, but the object is now considerably bluer.

According to Sandage (1971), SV 63 should be a variable of unknown type, but it was found constant by Carlson \& Sandage (1990). The star could correspond either to $\operatorname{V0016D}(V=22.60, V-I=1.19)$ or to $V 0017 D$ $(V=22.20, V-I=0.97)$ : both of them are close to the position given by Sandage in its identification map and both are constant within $0.1 \mathrm{mag}$. According to the GCVS coordinates (Samus 1995) the former should be the correct target, however, as we have already remarked in Papers I and III, these coordinates can be wrong by a few arcsec.

\section{Conclusion}

We have presented the results of the search for variable stars in Fields C and D of IC 1613. The light curves of 3297 stars were studied and 92 variable objects were detected, while previously only 8 variable stars were known in these fields. Our data do not confirm the variability of the stars $S V 50$ and $S V 63$ found by Sandage (1971). The detected objects have average $W h$ magnitude as faint as 23.2 mag. There are 34 Population I Cepheids, whose Period-Luminosity relation is plotted in Fig. 9. The objects are plotted using different symbols according to their pulsation modes. We also plot, just for illustrative purposes the $P-L$ relation derived in Paper III. This relation was determined by performing a least-squares fit to all the bona fide fundamental mode Cepheids in Fields A and $\mathrm{B}$ (55 stars, with the exclusion of the very long period object $V 2396 A$ with $P=140 \mathrm{~d}$ ).

The present paper concludes our search for variable stars in the nearby galaxy IC 1613 . We detected a total of 322 variable stars in our four fields, including 128 Population I Cepheids (with periods ranging from 0.65 to 140 days), $12 \mathrm{~W}$ Vir candidates, 10 eclipsing binaries and one nova. A future paper will discuss the properties of all Cepheids, including their Fourier parameters. It will also analyze in detail the $P-L$ relation obtained from all Cepheids present in this galaxy.

As regards the galaxy NGC 6822, we are presently reducing the images acquired with the Dutch telescope, and at the same time we are completing the observations with the WFI attached to the $2.2 \mathrm{~m}$ telescope at ESO. 
Acknowledgements. The authors wish to thank A. A. Cole for kindly supplying them with the multicolor frames of the central region of IC 1613. We are also grateful to an anonymous referee for the comments and suggestions that allowed us to improve the paper.

\section{References}

Antonello, E., Mantegazza, L., Fugazza, D., Bossi, M., \& Covino, S. 1999, A\&A, 349, 55 (Paper I)

Antonello, E., Mantegazza, L., Fugazza, D., \& Bossi, M. 1999, A\&A, 350, 797 (Paper II)

Antonello, E., Fugazza, D., Mantegazza, L., Bossi, M., \& Covino, S. 2000, A\&A, in press (Paper III)

Antonello, E., Fugazza, D., \& Mantegazza, L. 1999, A\&A, 356, L37

Beaulieu, J. P., \& Sasselov, D. D. 1997, in Variable Stars and the Astrophysical Returns of Microlensing Surveys, ed. R. Ferlet, J. P. Maillard, B. Raban (Éditions Frontières), 193 Carlson, G., \& Sandage, A. 1990, ApJ, 352, 587

Cole, A. A., Tolstoy, E., Gallagher, J. S., et al. 1999, AJ, 118, 1657 (CTG)

Davis, L. E. 1994, A Reference Guide to the IRAF/DAOPHOT Package, IRAF Programming Group, NOAO, Tucson
Freedman, W. L. 1988a, ApJ, 326, 691

Freedman, W. L. 1988b, AJ, 96, 1248 (FRE)

Fugazza, D., Mantegazza, L., Poretti, E., \& Antonello, E. 2000, IBVS, No. 4942

Kaluzny, J., Stanek, K. Z., Krockenberger, M., et al. 1998, AJ, 115,1016

Kaluzny, J., Mochejska, B. J., Stanek, K. Z., et al. 1999, AJ, 118,346

King, J. Y., Modjaz, M., \& Li, W. D., IAU Circ., No. 7287

Landolt, A. U. 1992, AJ, 104, 340

Samus, N. N. 1995, General Catalogue of Variable Stars, IV ed., vol. V, Moscow, Kosmosinform

Sandage, A. 1971, ApJ, 166, 13

Stetson, P. B. 1987, PASP, 99, 191

Stetson, P. B. 1996, PASP, 108, 851

Tody, D. 1993, in Astronomical Data Analysis Software and Systems II, ASP Conf. Ser., 52, ed. R. J. Hanisch, R. J. V. Brissenden, \& J. Barnes, 173

Udalski, A., Soszynski, I., Szymanski, M., et al. 1999, Acta Astron., 49, 473

Welch, D. L., Alcock, C., Allsman, R. A., et al. 1997, in Variable Stars and the Astrophysical Returns of Microlensing Surveys, ed. R. Ferlet, J. P. Maillard, B. Raban (Éditions Frontières), 205 